\title{
PREVALENCIA DE MALOCLUSIONES DENTALES EN ESCOLARES DE 12 AÑOS EN MONAY - CUENCA 2016
}

\section{PREVALENCE OF DENTAL MALOCLUSIONS IN SCHOOLCHILDREN OF 12 YEARS IN MONAY - CUENCA 2016}

\author{
Fajardo-Verdugo Juan. ${ }^{1 *}$, González-Campoverde Lorena. ${ }^{2}$ \\ ${ }^{1}$ Estudiante de la Facultad de Odontología de la Universidad Católica de Cuenca. Ecuador. \\ ${ }^{2}$ Catedrática de la Facultad de Odontología de la Universidad Católica de Cuenca.Ecuador. \\ *ffajardo28@gmail.com
}

\begin{abstract}
Resumen
OBJETIVO: El presente estudio tuvo como objetivo determinar la prevalencia de maloclusiones dentales en niños de 12 años en etapa escolar de la parroquia Monay en la ciudad de Cuenca-Ecuador. MATERIALES Y MÉTODOS: Este estudio epidemiológico fue de tipo descriptivo, observacional y corte transversal, la evaluación clínica bucal se realizó con la participación de 252 escolares, de los cuales 124 (49\%) representaron al sexo femenino y 128 (51\%) representaron al sexo masculino, presentando así una muestra equitativa de acuerdo al sexo, en éste estudio se incluyeron 3 escuelas fiscales y 2 escuelas particulares, de los cuales 136 escolares pertenecieron a colegios fiscales representando el $54 \%$ y 116 escolares pertenecieron a colegios particulares representando al $46 \%$. Se tomaron los datos con el paciente mirando de frente y usando espejos para el examen intraoral y de perfil para el examen extraoral con el fin de llegar a un diagnóstico presuntivo, todos los datos fueron registrados en la ficha epidemiológica. RESULTADOS: La prevalencia de maloclusiones fue del $85 \%$ del total de escolares, y el $15 \%$ presentaban normoclusión; de acuerdo al sexo las mujeres presentaron un $83 \%$ de maloclusiones frente a un $86 \%$ en los hombres, no hubo una relevancia significativa de maloclusiones según la gestión académica con un $84 \%$ en escuelas fiscales y un $85 \%$ en colegios particulares. CONCLUSIÓN: En este estudio epidemiológico se concluyó que existe una prevalencia significativa de maloclusiones.
\end{abstract}

Palabras clave: Maloclusión, sexo, escuelas, prevalencia.

\begin{abstract}
AIM: The objetive of this study was to determine the prevalence of dental malocclusions in 12 years old children in school stage at the neighborhood of Monay in the city of Cuenca-Ecuador. MATERIALS AND METHODS: It was a descriptive epidemiological, observational and retrospective cross-sectional study. 252 school age patients were involved in an oral clinical evaluation, of which $(49 \%)$ were female and 128 (51\%) were male. sex This represented a fair sample according to gender, this study involved three public schools and 2 private schools of which 136 students belonged to state schools representing $54 \%$ and 116 students belonged to private schools representing 46\%. The data was taken with the patient looking straight ahead and using mirrors for intraoral and extraoral profile for the examination in order to reach a presumptive diagnosis examination. All data was recorded in an epidemiological tab. RESULTS: The prevalence of malocclusion of all schoolchildren was $85 \%$, the percentage normooclusión was 15\%; according to gender women had $83 \%$ of malocclusions compared with $86 \%$ of men. There was also no significant relevance of malocclusions according to academic management, presented $84 \%$ in public schools and $85 \%$ in private schools. CONCLUSION: This epidemiological study concluded that there is a significant prevalence of malocclusions.
\end{abstract}

Key words: Malocclusion, sex, school, prevalence.

\section{INTRODUCCIÓN}

El presente tema de investigación se enmarcó en el área de la especialidad de Ortodóncia, ciencia dedicada a la prevención, tratamiento y corrección de las anomalías dentomaxilofaciales. Las maloclusiones se definen como la mal posición de las piezas dentarias y/o bases maxilares, en sus relaciones estáticas y dinámicas con sus piezas dentales adyacentes y antagonistas; además las relaciones de contacto no son las apropiadas entre la arcada superior e inferior. ${ }^{1}$ Las maloclusiones son la consecuencia de la adaptación orofacial a varios factores etiológicos locales o ambientales, los cuales generan diversas condiciones que varían desde 
alteraciones funcionales como la masticación, deglución, fonación, disfunción temporomandibular hasta la insatisfacción en la estética facial. La etiología es multifactorial, en ésta intervienen factores de predisposición genética o herencia y factores exógenos que pueden incluir desde un hábito bucal perjudicial hasta enfermedades, entre otras. ${ }^{2}$ Según la Organización Mundial De La Salud (OMS) "las maloclusiones constituyen la tercera enfermedad bucal de mayor prevalencia en las enfermedades a nivel mundial". ${ }^{3}$

Los tipos de maloclusiones se valoran según la clasificación universal de Angle y pueden tener modificaciones funcionales, esqueléticas, dentarias y de ATM. Angle, basado en estudios de cráneos en personas vivas, estableció los principios de oclusión y observó que el primer molar superior se halla bajo el contrafuerte lateral del arco cigomático, llamado "cresta llave" del maxilar superior y ésta relación es inalterable e hizo de ella la base para su clasificación, no se permitía una posición defectuosa de la dentición superior o del maxilar superior. En 1899, ideó un esquema simple y mundialmente aceptado, Angle introdujo la palabra "clase" para designar las relaciones mesiodistales de los dientes, las arcadas dentarias y los maxilares; que dependían de la perspectiva sagital de los primeros molares permanentes, a los que consideró como puntos fijos de referencia en la arquitectura cráneo facial. Este autor dividió las maloclusiones en tres grandes grupos: "Clase I, Clase II, Clase III.". ${ }^{4}$

Este tema de investigación se enfoca en el estudio de la prevalencia de maloclusiones en niños de 12 años de la parroquia Monay del Cantón Cuenca, pues al ser una enfermedad de alta prevalencia merece un estudio epidemiológico de la frecuencia de ésta en la población escolar, lo que permite en un futuro generar planes de salud bucodental orientados principalmente en la prevención.

\section{MATERIALES Y MÉTODOS}

Esta investigación fue un estudio de tipo epidemiológico, con un enfoque cuantitativo, observacional, transversal y descriptivo. La población fue de 252 escolares de 12 años de edad. ${ }^{5}$ Para realizar el estudio se solicitó el permiso de las instituciones educativas de la parroquia Monay mediante la autorización emitida por el distrito zonal 6 de educación, se les pidió autorización a los padres mediante el uso de un consentimiento informado y se les solicitó la aprobación de un asentimiento a los escolares para iniciar el examen clínico. Las condiciones para el examen fueron el operador sentado junto al paciente, se realizó el examen clínico con luz natural, el instrumental utilizado fue un espejo bucal y reglas milimetradas, se usaron todos los métodos de asepsia, antisepsia y las medidas de barreras de protección para el operador.

El examinador siguió las consecuentes recomendaciones, no tocar la boca del paciente con los dedos, mediante la inspección intraoral se ocuparon espejos bucales, se estableció el tipo de oclusión en base a la clasificación de Angle según

\begin{tabular}{lllllllllll}
\hline & \multicolumn{3}{l}{ FEMENINO } & \multicolumn{4}{c}{ MASCULINO } & \multicolumn{3}{c}{ TOTAL } \\
& FISCAL & PARTICULAR & FISCAL PARTICULAR & \\
\hline & $\mathbf{n}$ & $\%$ & $\mathbf{n}$ & $\%$ & $\mathbf{n}$ & $\%$ & $\mathbf{n}$ & $\%$ & $\mathbf{n}$ & $\%$ \\
MALOCLUSIÓN & 66 & 62 & 40 & 38 & 48 & 45 & 59 & 55 & 213 & 85 \\
NORMOCLUSIÓN & 12 & 67 & 6 & 33 & 10 & 48 & 11 & 52 & 39 & 15 \\
\hline
\end{tabular}

Tabla 1. Maloclusión en escolares de 12 años

las características de cada uno pudiendo ser clase I, clase II, clase II-1, clase II-2 y clase III, tomando en cuenta la clase molar y canina, se exploró el estado de salud de tejidos duros y blandos, así como la presencia de discrepancias dentales en los planos sagital, vertical y transversal, analizando: mordida abierta anterior y posterior, mordida cruzada anterior y posterior, sobremordida vertical, sobremordida horizontal, desviación de la línea media, mordida borde a borde. ${ }^{6-8,11,14}$ Para obtener el perfil anteroposterior se usaron los puntos glabela, subnasal y pogonión blando determinado el perfil facial; convexo, recto y cóncavo. ${ }^{9}$ Para obtener el perfil vertical se usaron los planos de Camper (Desde el borde inferior del ala de la nariz hasta el trago de la oreja.) y el plano mandibular (Desde el punto gonion hasta el punto gnation.) determinando los perfiles normodivergente, hiperdivergente e hipodivergente. ${ }^{10}$ El diagnóstico presuntivo se realizó mediante la observación visual clínica del rostro del paciente en sentido sagital y se asoció con las características de la oclusión en máxima intercuspidación. ${ }^{13,15}$

\section{RESULTADOS}

En el presente estudio participaron 252 escolares de 12 años de edad de los cuales $49 \%$ corresponden al sexo femenino y el $51 \%$ corresponden al sexo masculino, se incluyeron 3 escuelas fiscales y 2 escuelas particulares de los cuales 136 escolares pertenecieron a colegios fiscales representando el $54 \%$ y 116 escolares pertenecieron a colegios particulares representando al $46 \%$. En escuelas fiscales según el sexo, en el género femenino se presentaron 78 escolares que corresponde al $31 \%$, y en el género masculino el $23 \%$ representó a 58 escolares, mientras que, en las escuelas particulares, el género femenino fue de $18 \%$ que corresponde a 46 escolares y en el sexo masculino fue del $28 \%$ que corresponde a 70 escolares. $^{21,22}$

En la tabla 1 se observa que el $85 \%$ presentó maloclusión, con un mayor porcentaje en el sexo femenino en escuelas fiscales con el $62 \%$ y un menor porcentaje en escuelas particulares con el $38 \%$, para la determinación de las maloclusiones se valoró las alteraciones en sentido vertical, transversal y sagital.

En la tabla 2 se puede notar que la relación molar derecha en escuelas fiscales prevalece la clase I con el $77 \%$ y en las escuelas particulares con el $63 \%$; y la relación molar izquierda en escuelas particulares demostró una mayor 


\begin{tabular}{|c|c|c|c|c|c|c|c|c|c|c|c|c|c|c|c|}
\hline & & & $\begin{array}{rl}\mathrm{E} & \mathrm{LAC} \\
\mathrm{DE}\end{array}$ & $\begin{array}{l}\text { CIÓN } \\
\text { EREC }\end{array}$ & $\begin{array}{l}\mathrm{MO} \\
\mathrm{CHA}\end{array}$ & LAR & & & & & $\begin{array}{l}\text { ELAC } \\
\qquad I Z C\end{array}$ & $\begin{array}{l}\text { CIÓN } \\
\text { QUIE }\end{array}$ & $\begin{array}{l}\mathrm{N} M \mathrm{MO} \\
\mathrm{RDA}\end{array}$ & LAR & \\
\hline & CLA & $\begin{array}{l}\text { ASE } \\
\text { I }\end{array}$ & $\mathrm{CLA}$ & $\begin{array}{l}\text { ASE } \\
\text { II }\end{array}$ & & $\begin{array}{l}\text { ASE } \\
\text { III }\end{array}$ & & A. & CLA & $\begin{array}{l}\text { ASE } \\
\text { I }\end{array}$ & CLA & $\begin{array}{l}\text { ASE } \\
\text { II }\end{array}$ & CLA & ASE & N.A. \\
\hline & $\mathbf{n}$ & $\%$ & $\mathbf{n}$ & $\%$ & $\mathbf{n}$ & $\%$ & $\mathbf{n}$ & $\%$ & $\mathbf{n}$ & $\%$ & $\mathbf{n}$ & $\%$ & $\mathbf{n}$ & $\%$ & n $\%$ \\
\hline FISCAL & 60 & 77 & 13 & 17 & 3 & 4 & 2 & 3 & 54 & 69 & 16 & 21 & 6 & 8 & 23 \\
\hline PARTICULAR & 29 & 63 & 12 & 26 & 4 & 9 & 1 & 2 & 28 & 61 & 12 & 26 & 6 & 13 & 00 \\
\hline TOTAL & 89 & 72 & 25 & 20 & 7 & 6 & 3 & 2 & 82 & 66 & 28 & 23 & 12 & 10 & 22 \\
\hline
\end{tabular}

Tabla 2. Relación Molar de Angle según el sexo Femenino y la Gestión Académica.

\begin{tabular}{|c|c|c|c|c|c|c|c|c|c|c|c|c|c|c|}
\hline & \multicolumn{7}{|c|}{$\begin{array}{l}\text { RELACIÓN MOLAR } \\
\text { DERECHA }\end{array}$} & \multicolumn{7}{|c|}{$\begin{array}{l}\text { RELACIÓN MOLAR } \\
\text { IZQUIERDA }\end{array}$} \\
\hline & \multicolumn{2}{|c|}{$\underset{I}{\text { CLASE }}$} & \multicolumn{2}{|c|}{$\underset{\text { II }}{\text { CLASE }}$} & \multicolumn{2}{|c|}{$\begin{array}{c}\text { CLASE } \\
\text { III }\end{array}$} & N.A. & \multicolumn{2}{|c|}{$\underset{I}{\text { CLASE }}$} & \multicolumn{2}{|c|}{$\underset{\text { II }}{\text { CLASE }}$} & \multicolumn{2}{|c|}{$\begin{array}{l}\text { CLASE } \\
\text { III }\end{array}$} & \multirow{2}{*}{$\begin{array}{l}\text { N.A. } \\
\text { n } \%\end{array}$} \\
\hline & $\mathbf{n}$ & $\%$ & $\mathrm{n}$ & $\%$ & $\mathbf{n}$ & $\%$ & n \% & $\mathbf{n}$ & $\%$ & $n$ & $\%$ & n & $\%$ & \\
\hline FISCAL & 37 & 64 & 12 & 21 & 8 & 14 & 12 & 36 & 62 & 14 & 24 & 7 & 12 & 12 \\
\hline PARTICULAR & 41 & 59 & 14 & 20 & 10 & 14 & 57 & 41 & 59 & 15 & 21 & 10 & 14 & 46 \\
\hline TOTAL & 78 & 61 & 26 & 20 & 18 & 14 & 65 & 77 & 60 & 29 & 23 & 17 & 13 & 54 \\
\hline
\end{tabular}

Tabla 3. Relación Molar de Angle según el sexo Masculino y la Gestión Académica.

prevalencia de clase I con el $69 \%$ y en las escuelas particulares mostró un $61 \%$; y en menor porcentaje los que no presentaban relación molar tanto en escuelas fiscales como particulares.

En la tabla 3 se indica que la relación molar derecha en escuelas fiscales presentó un mayor porcentaje en la clase I con el $64 \%$ y en la relación molar izquierda el $62 \%$; en tanto que en las escuelas particulares se determinó el $59 \%$ en la clase I molar derecha e izquierda y en menor porcentaje los que no presentaban relación molar tanto en escuelas fiscales como particulares.

En la tabla 4 se indica que la relación canina según Angle en escuelas fiscales la clase I en el lado derecho presentó un mayor porcentaje con el $56 \%$ y en el lado izquierdo el $42 \%$; en escuelas particulares se presentó mayor porcentaje en la clase I del lado derecho con un $39 \%$ y en el lado izquierdo un $35 \%$; pero también se notó un número

\begin{tabular}{|c|c|c|c|c|c|c|c|c|c|c|c|c|c|c|c|}
\hline & \multicolumn{8}{|c|}{$\begin{array}{c}\text { RELACIÓN CANINA } \\
\text { DERECHA }\end{array}$} & \multicolumn{6}{|c|}{$\begin{array}{l}\text { RELACIÓN CANINA } \\
\text { IZQUIERDA }\end{array}$} & \multirow[b]{2}{*}{ N.A. } \\
\hline & \multicolumn{2}{|c|}{$\underset{I}{\text { CLASE }}$} & \multicolumn{2}{|c|}{$\underset{\text { II }}{\text { CLASE }}$} & \multicolumn{2}{|c|}{$\begin{array}{c}\text { CLASE } \\
\text { III }\end{array}$} & \multicolumn{2}{|c|}{ N.A. } & \multicolumn{2}{|c|}{$\begin{array}{c}\text { CLASE } \\
I\end{array}$} & \multicolumn{2}{|c|}{$\underset{\text { II }}{\text { CLASE }}$} & \multicolumn{2}{|c|}{$\begin{array}{c}\text { CLASE } \\
\text { III }\end{array}$} & \\
\hline & $\mathrm{n}$ & $\%$ & $\mathbf{n}$ & $\%$ & $\mathrm{n}$ & $\%$ & $\mathrm{n}$ & $\%$ & $\mathrm{n}$ & $\%$ & $n$ & $\%$ & $n$ & $\%$ & n \% \\
\hline FISCAL & 44 & 56 & 8 & 10 & 6 & 8 & 20 & 26 & 33 & 42 & 9 & 12 & 5 & 6 & 314 \\
\hline PARTICULAR & 18 & 39 & 6 & 13 & 2 & 4 & 20 & 43 & 16 & 35 & 5 & 11 & 3 & 7 & 224 \\
\hline TOTAL & 62 & 50 & 14 & 11 & 8 & 6 & 40 & 32 & 49 & 40 & 14 & 11 & 8 & 6 & $\begin{array}{ll}53 & 4\end{array}$ \\
\hline
\end{tabular}

Tabla 4. Relación Canina de Angle según el sexo Femenino y la Gestión Académica.

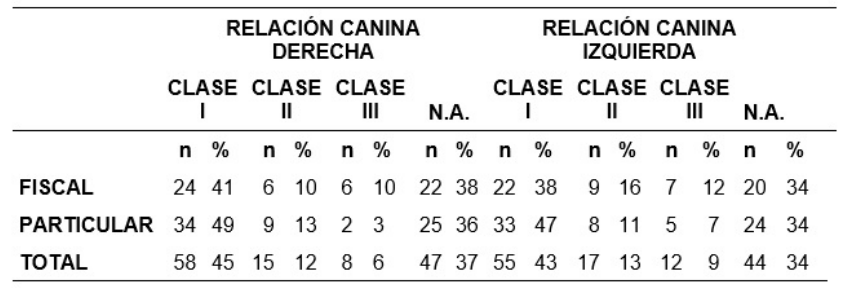

Tabla 5. Relación Canina de Angle según el sexo Masculino y la Gestión Académica.

\begin{tabular}{|c|c|c|c|c|c|c|c|c|c|c|c|c|c|c|c|c|}
\hline & & & & FEME & ENII & NO & & & & & & & & Ascul & LINC & \\
\hline & & ${ }_{1}^{A S B}$ & & $\begin{array}{l}\text { LASE } \\
\text { II-1 }\end{array}$ & & $\begin{array}{l}\text { LASE } \\
\text { II-2 }\end{array}$ & & $\begin{array}{l}\text { LASE } \\
\text { III }\end{array}$ & & $\underset{I}{\text { LASE }}$ & & $\begin{array}{l}\text { LASE } \\
\text { II-1 }\end{array}$ & & $\begin{array}{l}\text { CLASE } \\
\text { II-2 }\end{array}$ & & $\begin{array}{l}\text { LASE } \\
\text { III }\end{array}$ \\
\hline & $\mathbf{n}$ & $\%$ & $\mathbf{n}$ & $\%$ & $\mathbf{n}$ & $\%$ & $\mathrm{n}$ & $\%$ & $\mathbf{n}$ & $\%$ & $\mathbf{n}$ & $\%$ & $\mathbf{n}$ & $\%$ & $\mathrm{n}$ & $\%$ \\
\hline FISCAL & 53 & 68 & 16 & 21 & 3 & 4 & 6 & 8 & 26 & 45 & 16 & 28 & 5 & 9 & 11 & 19 \\
\hline $\begin{array}{l}\text { PARTICULA } \\
\mathbf{R}\end{array}$ & 25 & 54 & 13 & 28 & 2 & 4 & 6 & 13 & 37 & 53 & 17 & 24 & 4 & 6 & 12 & 17 \\
\hline TOTAL & 78 & 63 & 29 & 23 & 5 & 4 & 12 & 10 & 63 & 49 & 33 & 26 & 9 & 7 & 23 & 18 \\
\hline
\end{tabular}

Tabla 6. Prevalencia de la Clase Esqueletal mediante Diagnostico Presuntivo según el sexo y la Gestión Académica.

elevado de escolares que no presentaban relación en escuelas particulares en el lado izquierdo con el $48 \%$ y en el lado derecho con el $43 \%$; y con un menor porcentaje la clase III tanto en escuelas fiscales como particulares.

En la tabla 5 se indica que la relación canina según Angle mostró que, en escuelas particulares la clase I del lado derecho presentó un mayor porcentaje con el $49 \%$ en relación con el lado izquierdo que obtuvo el $47 \%$; en escuelas fiscales se presentó mayor porcentaje en la clase I del lado derecho con un $41 \%$ y en el lado izquierdo un $38 \%$; pero en este caso se mostró un elevado porcentaje de los que no presentaban relación canina en el lado derecho en escuelas fiscales con el $38 \%$ y en el lado izquierdo en escuelas fiscales y particulares con el $34 \%$, con un menor porcentaje la clase III tanto en escuelas fiscales como particulares.

En la tabla 6 se analizó la clase esqueletal mediante diagnóstico presuntivo, en donde se presentó un mayor porcentaje de clase I en el sexo femenino tanto en escuelas fiscales con el $68 \%$ como en escuelas particulares con el $54 \%$ y en menor porcentaje la clase II-2 tanto en escuelas fiscales como particulares.

\section{Discusión}

La maloclusión es una enfermedad multifactorial de origen genético y ambiental que afecta la estética del paciente además de la fonética, la masticación y la deglución siendo importante su temprano diagnóstico y tratamiento para evitar enfermedades como la caries y enfermedad periodontal.

En estudios realizados por Dimberg y cols, ${ }^{19}$ en el año 2015 en una muestra de 277 escolares la prevalencia de maloclusiones fue del $71 \%$ en niños de 11.5 años, estudios realizados 
por Narayaman y cols, ${ }^{26}$ en el año 2016 presentaron el $83.3 \%$ de maloclusiones y según Pinchera y cols, ${ }^{23}$ en el año 2016 en una muestra de 46 escolares presentaron una prevalencia de maloclusiones del $91.3 \%$, así mismo Mahesh y cols, ${ }^{24}$ en el año 2005 en una muestra de 482 escolares presentaron un total de maloclusiones de $96.3 \%$. El reciente estudio presentó un $85 \%$ de maloclusiones en una muestra de 252 escolares similar a investigaciones realizadas. ${ }^{9,12,20,27}$

Según Jordao y cols, ${ }^{25}$ en el año 2015 con una muestra de 2692 escolares presentaron una maloclusion de $40.2 \%$ en mujeres y $40 \%$ en hombres de los cuales presentaron un $30.3 \%$ de maloclusiones en escuelas privadas y un $44.1 \%$ en escuelas públicas; estudio similar de Narayaman y cols, ${ }^{26}$ en el año 2016 reportó una prevalencia de maloclusiones del $54.1 \%$ en hombres y $45.9 \%$ en mujeres. Este estudio según la gestión académica en escuelas fiscales en el sexo femenino se presentó un $62 \%$ de maloclusiones y un $48 \%$ en el sexo masculino y en escuelas privadas en el sexo femenino un $38 \%$ de maloclusiones y un $55 \%$ en el sexo masculino presentando una diferencia no significativa al igual que estudios citados. ${ }^{16,17}$

Estudios realizados por Rodríguez ${ }^{20}$ en el año 2016 con una muestra de 352 escolares demostró que $69 \%$ de estudiantes presentaron clase I, el $19 \%$ presentaron clase II y el $11.9 \%$ con clase III; estudios similares realizados por Villasana y cols, ${ }^{22}$ en el año 2013 en una muestra de 76 escolares en relación a la clase molar derecha presentó, clase I el $61.8 \%$, clase II $23.7 \%$ y clase III $14.5 \%$, respecto al lado izquierdo, se identificó clase I $61.8 \%$, clase II $19.7 \%$ y clase III $18.4 \%$. El presente estudio similar a los anteriores demostró que en escolares de acuerdo al sexo y a la gestión académica en el sexo femenino en escuelas fiscales y particulares la relación molar derecha presentó la clase I $72 \%$, clase II $20 \%$ y clase III $6 \%$ y los que no registraban $2 \%$, la relación molar izquierda fue del $66 \%$ para la clase I, el $23 \%$ para la clase II, el $10 \%$ para la clase III y los que no registraban relación $2 \%$. En relación al sexo masculino la clase molar derecha, clase I $61 \%$, clase II $20 \%$, clase III $14 \%$ y los que no registraban $5 \%$ y la relación molar izquierda la clase I $60 \%$, clase II $23 \%$, clase III $13 \%$ y los que no registraban relación molar $4 \%$ similar a estudios realizados en investigaciones citadas anteriormente que se encuentran en el rango en la clase molar I, II y III. $^{22}$

Según estudios realizados por Narayanan y cols, ${ }^{26}$ en el año 2016 presentaron maloclusión de clase I $69.8 \%$; clase II $9.3 \%$ y clase III $4.1 \%$; el $8.85 \%$ clase II división 1; y el $0.5 \%$ clase II división 2; en estudios realizados por Fuentes y cols, ${ }^{21}$ la prevalencia fue en la clase I $39 \%$; clase II $12 \%$; clase II subdivisión $17 \%$ y la clase III $36 \%$. En este estudio en relación al sexo y a la gestión académica se demostró la clase I $63 \%$; la clase II división 1 el $26 \%$; la clase II división 2 el $7 \%$; y la clase III $18 \%$; constando en el rango de estudios realizados anteriormente. ${ }^{27}$ Mientras que el estudio realizado por Fuentes y cols, ${ }^{19}$ en el año 2016 con una muestra de 390 escolares muestran valores menores en la clase I y mayores en la clase III en comparación al presente estudio.

\section{Referencias}

1 Ministerio de salud pública del Ecuador. Protocolos odontológicos. Ed. Dirección nacional de normatización. 2014. 1(1) 179-198. Documento disponible

2 Proffit WR, Fields HW, Sarver DM. Ortodoncia contemporánea: Teoría y práctica. Madrid. Ed. Elsevier. 2001.12(1); 2-247

3 Pan American health organization. Health in the Americas. Washington DC: Paho; 1998. p. 413-27.

4 Ugalde F. Clasificación de la maloclusión en los planos anteroposterior, vertical y transversal. Revista ADM.2007, 1(3): 97-109

5 Villavicencio E. El tamaño muestral en tesis de postgrado. ¿cuántas personas debo encuestar? Revista Researchgate [internet].2011. [citado el 1 de agosto del 2016].2011;1(1);1-4

6 Campos A, Rehabilitación oral y oclusal. en: Oclusión y Articulación Temporomandibular. Vol. 1. Madrid, Harcout. 2000. p (1). 3-1

7 Arroyo YM, Morera MH. Maloclusiónes en niños de 8 a 12 años y percepción de los padres de familia de la necesidad de una interconsulta con el ortodoncista a temprana edad. Revista Científica Odontológica [Internet]. [Citado 01 De agosto 2016]. 2012; 8 (2):19-24

8 Floria G Virtual Journal Of Orthodontics. [Internet] 2000 [citado 30 de agosto del 2016] 2000; 117:444-452

9 Sánchez D, Sánchez A. Características faciales y anomalías de malposición dentarias más comunes en hombres de 18 a 2 años de la II zona naval en galápagos. Revista Latinoamericana De Ortodoncia Y Odontopediatría: 2010; 1(1); 1-15.

10 Castillo C, Sosa J, Ríos D. Frecuencia de mordida abierta anterior en escolares del primer noveno grado y sus factores asociados. Revista Pediatr: 2012; 39(2); 103-106. Documento disponible

11 Ferreira V, Martins F. Oclusión y equilibrio de los dientes, clasificación de las maloclusiónes. en: Ferreira V, Hecht M. Ortodoncia diagnóstico y planificación clínica. Vol. 1. Sao Paulo. Artes Médicas Latinoamérica; 2002. p. 74-114.

12 Kiparta M, Kerosuo M, Nystro M. Orthodontic treatment need from eight to 12 years of age in an early treatment oriented public health care system: A Prospective Study. Revista. The Angle Orthodontist: 2005; 75, (3); 344-349. Documento disponible

13 Gregoret J, Tuber E. Ortodoncia y cirugía ortognatica diagnóstico y planificación. en: Examen Bucodental. Barcelona 1997. p. 1(2); 49-61-460.

14 Saadia M, Jeffrey H. Atlas de ortopedia dentofacial durante el crecimiento, Barcelona: Espaxs, S. A. 2000, cap 1 (1): 7. Documento disponible

15 Uribe G, Fundamentos de odontología ortodoncia teoría y clínica. en: Análisis de los arcos dentales. Vol.1.Colombia. 
Corporación para investigaciones biológicas. p. 81-432 .

Recibido: 10 de Enero de 2016

Documento disponible

16 Millan T, Katagiri M, Perez H, Tejada E. Causística de maloclusiónes clase I, clase II y clase III según Angle en el departamento de ortodoncia de la UNAM. Revista Medigraphic Artemisa: 2007; 11(4); 175-80. Documento disponible

17 Filho O, Ferrari J, Okada T. Dental arch dimensions in class II division 1 malocclusions with mandibular deficiency. Revista The Angle Orthodontist: 2008; 78, (2); 466-474.

18 Das UM, Beena JP, Azher U. Oral health stadus of 6 and 12 year old school going children in Bangalore City: an epidemiological study. J Indian Soc Pdo Prev Dent.[Internet]. [Citado El 10 De agosto Del 2016], 2009; 1(27)6-8. . Documento disponible

19 Dimberg L, Lennartsson B, Arnrup K, Bondemark L. Prevalence and change of malocclusions from primary to early permanent dentition: a longitudinal study. Revista Angle Orthodontist. 2015;85(5);728-735. Documento disponible

20 Rodríguez J. Prevalencia de maloclusión y necesidad de tratamiento ortodóntico en escolares de 12 años de instituciones educativas públicas-Trujillo 2015. Rev. Dspace. Unitru. 2016; 1(2):1-16.

21 Fuentes C, Muñoz F, San Martin N, Oliva C. Necesidad de tratamiento ortodóncico según índice DAI y Angle en adolescentes de 1 a 13 años de la comuna de Curanilahue en el año 2014. Revista Int. J. Med. Surg. Sci., 2016.3 (2):829837.

22 Villasana P, Clarke E, Hernández T. Prevalencia de maloclusiónes en niños de la primaria "6 de abril" en Hermosillo, Sonora, México. Revista Amop, 2013, 25(1):102-107.

23 Pincheira C, Thiers L. Bravo S, Olave C. Articulo prevalencia de maloclusiónes en escolares de 6 y 12 años de Choshuenco - Neltume, Chile. Int. J. Med. Surg. Sci., 2016; 3(2):829-837.

24 Mahesh KP, Joseph T, Varma RB, Jayanthi M. Oral health status of 5 years and 12 years school going children in Chennai City - an epidemiological study. J Indian Soc Pedo Prev Dent [Internet] [Citado el 8 de agosto del 2016]. 2005; 1 (1)17-22. Documento disponible

25 Jordao Ml, Vasconcelos D, Moreira R, Freire M. Individual and contextual determinants of maloclusión in 12 -year-old schoolchildren in a Brazilian City. Braz Oral Res [internet]. 2015; 29(1);2-8.

26 Narayanan R, Jeseem M, Kumar T. Prevalence of maloclusión among 10 - 12 years old schoolchildren in Kozhikode district, Kerala: an epidemiological Study. Editorial International Journal Of Clinical Pediatric Dentistry, 2016;9(1):5055

27 Candido V, Cortellazzi K, Vazquez L, Ambrosano G. Fatores individuais e contextuais asociados a ma oclusao em criancas brasileiras. Rev Saúde Pública 2013; 47(3):1-11. 
